\title{
Elastic proton-deuteron scattering at intermediate energies
}

\author{
A. Ramazani-Moghaddam-Arani ${ }^{1,2}$ * H.R. Amir-Ahmadi ${ }^{1}$, A.D. Bacher ${ }^{3}$, C.D. Bailey ${ }^{3}$, \\ A. Biegun ${ }^{1}$, M. Eslami-Kalantari ${ }^{1}$, I. Gašparić ${ }^{4}$, L. Joulaeizadeh ${ }^{1}$, N. Kalantar-Nayestanaki ${ }^{1}$, \\ St. Kistryn ${ }^{5}$, A. Kozela ${ }^{6}$, H. Mardanpour ${ }^{1}$, J.G. Messchendorp ${ }^{1}$ Of A.M. Micherdzinska ${ }^{7}$, \\ H. Moeini ${ }^{1}$, S.V. Shende ${ }^{1}$, E. Stephan ${ }^{8}$, E.J. Stephenson ${ }^{3}$, and R. Sworst ${ }^{5}$ \\ ${ }^{1}$ KVI, University of Groningen, Groningen, The Netherlands \\ 2 Department of Physics, Faculty of Science, University of Kashan, Kashan, Iran \\ 3 Indiana University Cyclotron Facility, Indiana, USA \\ 4 Rudjer Bošković Institute, Zagreb, Croatia \\ 5 Institute of Physics, Jagiellonian University, Cracow, Poland \\ 6 Henryk Niewodniczański, Institute of Nuclear Physics, Cracow, Poland \\ 7 University of Winnipeg, Winnipeg, Canada and \\ 8 Institute of Physics, University of Silesia, Katowice, Poland
}

(Dated: October 22, 2018)

\begin{abstract}
Observables in elastic proton-deuteron scattering are sensitive probes of the nucleon-nucleon interaction and three-nucleon force effects. The present experimental data base for this reaction is large, but contains a large discrepancy between data sets for the differential cross section taken at $135 \mathrm{MeV} /$ nucleon by two experimental research groups. This paper reviews the background of this problem and presents new data taken at KVI. Differential cross sections and analyzing powers for the ${ }^{2} \mathrm{H}(\vec{p}, d) p$ and $\mathrm{H}(\vec{d}, d) p$ reactions at $135 \mathrm{MeV} /$ nucleon and $65 \mathrm{MeV} /$ nucleon, respectively, have been measured. The data differ significantly from previous measurements and consistently follow the energy dependence as expected from an interpolation of published data taken over a large range at intermediate energies.
\end{abstract}

PACS numbers: 21.30.-x, 21.45.+v, 24.70.+s, 25.45.De

The nucleon-nucleon potential (NNP) has been studied extensively by investigating the properties of bound nuclear systems, and, in more detail, via a comparison of high-precision two-nucleon scattering data with modern potentials based on the exchange of bosons [1, 2, 3]. A few of the modern NNPs were facilitated by a partialwave analysis (PWA), that provides a nearly modelindependent analysis of the available scattering data [4]. The modern NNPs reproduce the world data base with a reduced chi-square close to one and have, therefore, been accepted as high-quality benchmark potentials. The precision of modern NNPs has given confidence to study in detail the three-nucleon potential (3NP) which was already predicted in 1939 by Primakoff and Wilson [5]. Compelling evidence of $3 \mathrm{NP}$ effects came from various recent theoretical and experimental studies. For example, for light nuclei, Green's function Monte-Carlo calculations employing the high-quality NNPs clearly underestimate the experimental binding energies 2], and, therefore, show that NNPs are not sufficient to describe the three-nucleon and heavier systems accurately. In the last decade, high-precision data at intermediate energies in elastic $N d$ and $d N$ scattering 6, 7, 8, 9, 10, 11, 12, 13, $14,15,16,17,18,19,20,21,[22,23]$ for a large energy range together with rigorous Faddeev calculations 24]

\footnotetext{
*Electronic address: ramazani@kvi.nl

${ }^{\dagger}$ Electronic address: messchendorp@kvi.nl
}

for the three-nucleon system have proven to be a sensitive tool to study the 3NP. In particular, a large sensitivity to $3 \mathrm{NP}$ effects exists in the minimum of the differential cross section [25, 26]. Precision data for a large energy interval for the differential cross section and analyzing power came from recent experimental studies at KVI 7, 8, 9], RIKEN [10] and RCNP [12]. All these experiments had one common energy of $135 \mathrm{MeV} /$ nucleon. Strikingly, the cross sections obtained at KVI were found to be significantly larger than those measured at RIKEN and at RCNP. The KVI data show significant deviation from predictions of state-of-the-art Faddeev calculations incorporating modern NNPs and 3NPs at this energy, whereas the results obtained at RIKEN and RCNP imply that the cross section can be described reasonably well exploiting the same potentials.

This paper presents the results of a new measurement of the differential cross sections of the reaction ${ }^{2} \mathrm{H}(\vec{p}, d) p$ at a proton-beam energy of $135 \mathrm{MeV}$, taken to provide additional data at $135 \mathrm{MeV} /$ nucleon. These results are compared with the previously published data taken at intermediate energies. The data are obtained at KVI using a new experimental equipment, Big Instrument for Nuclear-polarization Analysis (BINA). Also, systematic uncertainties were checked by measuring the inverse reaction $\mathrm{H}(\vec{d}, d) p$ at a deuteron-beam energy of $65 \mathrm{MeV} /$ nucleon using the same experimental setup and analysis methods. The differential cross section at this energy is well-known [21] and can, therefore, be exploited 
to verify independently the read-out and analysis procedure, the applied detector inefficiencies, and the beamcurrent measurement. For both experiments, polarization observables have also been measured. This allows to check some of the aspects of the experiment and the analysis procedure, excluding the absolute normalization, by comparing our results with data from the literature. Important to note is that the previously published KVI data were obtained using the Big-Bite Spectrometer, which was located at a different beam line and which used a completely different analysis framework, target, read-out and data-acquisition system.

BINA is a setup with a nearly $4 \pi$ geometrical acceptance and has been used in various few-nucleon scattering experiments to measure the scattering angles and energies of protons and deuterons with the possibility for particle identification. The detector is composed of a forward and a backward part. The forward part consists of a Multi-Wire Proportional Chamber (MWPC) 27] and a segmented hodoscope of vertically-placed thin scintillators with a thickness of $2 \mathrm{~mm}$ followed by ten horizontally-placed scintillators with a thickness of $12 \mathrm{~cm}$ each. The thick scintillators were mounted in a cylindrical shape, thereby, pointing to the target. The thickness of these scintillators is sufficient to stop all the protons and deuterons originating from the processes described in this paper. The detection efficiency of the MWPC was obtained by using an unbiased and nearly backgroundfree data sample of elastically-scattered deuterons and was found to be typically $97 \pm 1 \%$. The backward part has 149 phoswich scintillators covering polar angles between $40^{\circ}$ to $165^{\circ}$ with a nearly-full azimuthal coverage.

The polarized proton beams from the AGOR accelerator impinged on a liquid-deuterium [28] target with a thickness of $3.85 \mathrm{~mm}$, with an uncertainty of $5 \%$, which was mounted in the center of the backward part of BINA. The target cell is made of high-purity aluminum to optimize the thermal conductivity and the windows are covered by a transparent foil of Aramid with a thickness of $4 \mu \mathrm{m}$. The cell was operating under a pressure of 250 mbar and a temperature of $19 \mathrm{~K}$. For the deuteron beam, a solid organic $\mathrm{CH}_{2}$ target was used for the $\mathrm{H}(\vec{d}, d) p$ experiment with an effective thickness of $13.75 \pm 0.24 \mathrm{mg} / \mathrm{cm}^{2}$. The beam current was typically $15 \mathrm{pA}$ and was monitored continuously during the experiment via a Faraday cup at the end of the beam line. The current meter was calibrated using a precision current source with an uncertainty of about $2 \%$.

For this experiment, events were selected in which deuterons were detected in the forward part in coincidence with a scattered proton in the backward part of BINA. The efficiency of the coincidence hardware trigger was determined from a data sample obtained from a minimum-bias trigger and found to be $98 \pm 1 \%$. In the analysis, a large part of the background, pre-dominantly from the break-up reactions, was reduced by verifying that the forward and backward particles determined the same scattering plane within a limit of $\pm 20^{\circ}$. Figure 1

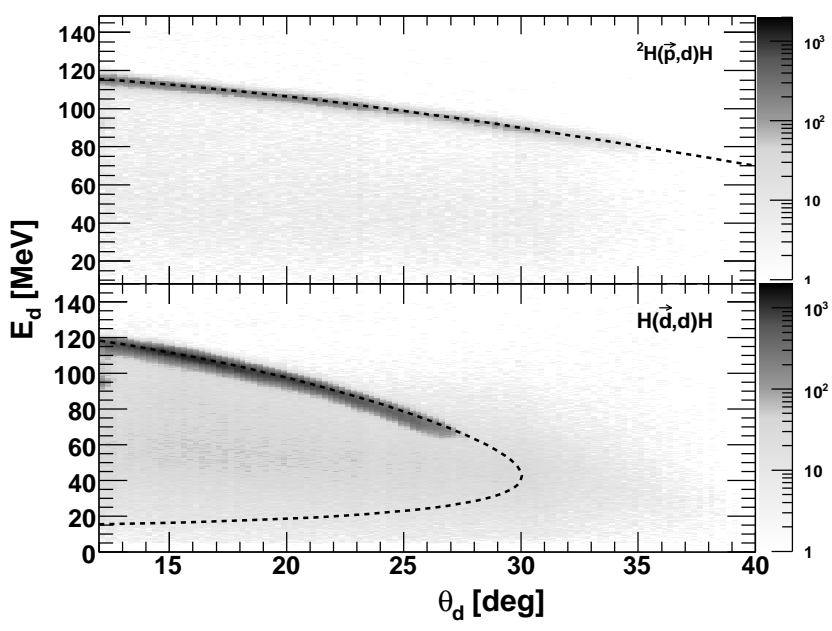

FIG. 1: The figure depicts the correlation between the scattering angle and the deposited energy for events registered in the forward part of BINA. The top panel corresponds to data taken using a proton beam with an energy of $135 \mathrm{MeV}$ impinging on a liquid-deuterium target. The bottom panel shows data taken with a deuteron beam of $130 \mathrm{MeV}$ impinging on a solid $\mathrm{CH}_{2}$ target. The dashed lines represent the expected kinematical correlation for a scattered deuteron in the elastic proton-deuteron reaction.

shows the correlation between the scattering angle of particles and their deposited energy in the forward part of BINA for both incident energies. The reactions ${ }^{2} \mathrm{H}(\vec{p}, d) p$ and $\mathrm{H}(\vec{d}, d) p$ can clearly be identified in the top and bottom panel, respectively. It can be seen that for both reactions, the forward-scattered deuterons have similar energies; thus the check against lower-energy data is relevant at $135 \mathrm{MeV} /$ nucleon. Peak sums were obtained by transforming the forward energy spectra to the center-of-mass system, reproducing these spectra with the sum of a Gaussian and a polynomial function of energy, and using for the peak sum the integral of the Gaussian function.

Part of the events away from the loci in Fig. 11 corresponds to elastically-scattered deuterons which undergo a hadronic interaction inside the scintillators. These events cannot be separated easily from the background from the break-up reaction. The contribution of particles undergoing hadronic interactions was obtained by analyzing part of the data for which stringent cuts on the scattering angle and energy of the backward scattered particles were applied. Since these cuts removed essentially all break-up background, events which fell outside the full energy peak were interpreted as the fraction of the elastic events that comprised the hadronic interaction tail below the range of integration of the Gaussian peak. The total fraction of events which suffer from a hadronic interaction is about $16 \%$ with an uncertainty of $2 \%$.

Although the emphasis of this work is to obtain differential cross sections in elastic proton-deuteron 


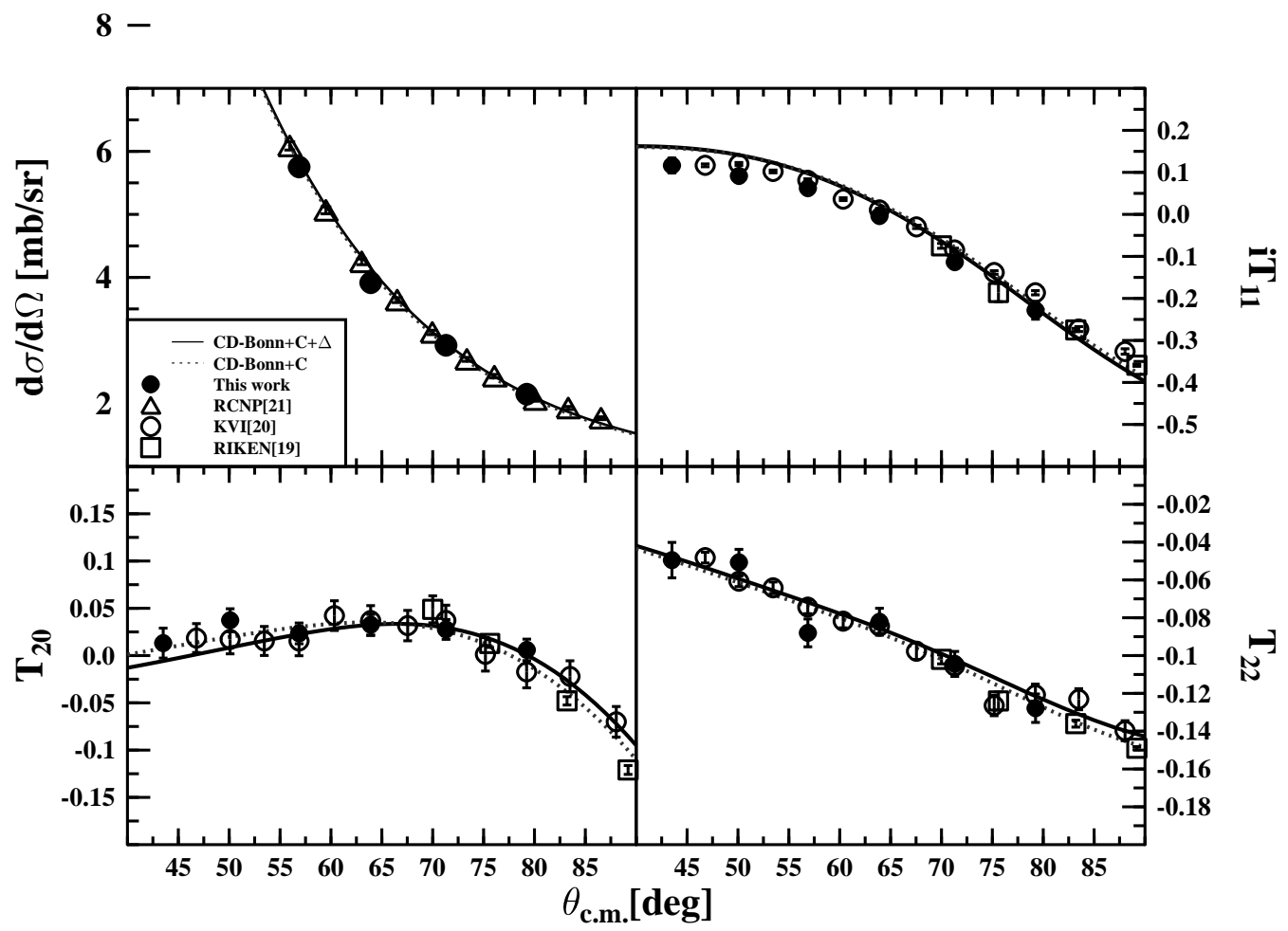

FIG. 2: The differential cross section, vector and tensor analyzing powers of the elastic $\vec{d}+p$ reaction at $E_{d}^{l a b}=130 \mathrm{MeV}$ as a function of the center-of-mass angle, $\theta_{c . m}$. In each panel, filled circles are data from this work. Only statistical uncertainties are shown for each point. The open triangles in top-left panel represent cross section data taken at RCNP [21]. The open circles and open squares in the other panels are analyzing power data taken at KVI [20] and at RIKEN [19], respectively. Note that we do not present differential cross sections for $\theta_{c m}<55^{\circ}$. At these angles, an increasing fraction of backward-scattered protons are undetected due to their small energy and the amount of material between the target and the detector. The solid lines represent the results of a coupled-channel calculation by the Hannover-Lisbon theory group and are based on the CDBonn potential including the Coulomb interaction and an intermediate $\Delta$-isobar. The results of a similar calculation, however, excluding the $\Delta$-isobar, are shown as dashed lines.

scattering, we have measured the various polarization observables for these reactions as well. A comparison with the existing and well-established world data base of vector and tensor analyzing powers allows us to verify the quality of the data. Beams of vector-polarized protons or vector and tensor-polarized deuterons were produced in an atomic-beam-type ion source. The polarization of the beams was measured using a Lamb-shift polarimeter (LSP) in the low-energy beam line and by an in-beam polarimeter (IBP) which was installed at the high-energy beam line after acceleration. More information on the operation of the IBP and LSP can be found in Refs. 29, 30]. During the experiments discussed in this paper, the beam polarization was typically $65 \%$ of the maximally-allowed theoretical values. The analyzing powers of the elastic $\vec{d}+p$ and $\vec{p}+d$ reactions were obtained by studying the dependence of the differential cross section on the azimuthal scattering angle of the deuterons in the final state. An equivalent data analysis has been reported and published in Ref. [20].

Figure 2 summarizes the measured cross sections and analyzing powers with only statistical errors for the $\mathrm{H}(\vec{d}, d) p$ reaction at a beam energy of $65 \mathrm{MeV} /$ nucleon and as a function of the center-of-mass angle, $\theta_{c . m}$. The systematic uncertainty of the measured differential cross section and analyzing powers is estimated to be $4 \%$ and $3 \%$, respectively. The data of this experiment are shown as filled circles and are compared with published cross section data taken in the past at KVI and elsewhere. The new results are in good agreement with the previously published data and, thereby, demonstrate that the experimental setup and the data-analysis procedure are well understood. The solid lines in Fig. 2 are the result of a rigorous Faddeev calculation by the HannoverLisbon theory group. In this calculation, the $\Delta$-isobar excitation mediates an effective $3 \mathrm{NP}$ with prominent Fujita-Miyazawa and Illinois ring-type contributions. These contributions are based on the exchange of $\pi, \rho$, $\omega$, and $\sigma$ mesons. For the dashed lines, the $\Delta$-isobar degree of freedom was switched off. The difference between the solid and dashed lines represents, therefore, a three-nucleon force effect within the framework of this model. Note that at this energy, the data are very well described by the state-of-the art calculations.

Figure 3 shows our results (filled circles) for the vector analyzing power (top panel) and the differential cross section (bottom panel) of the ${ }^{2} \mathrm{H}(\vec{p}, d) p$ reaction at a beam energy of $135 \mathrm{MeV}$ and as a function of the 


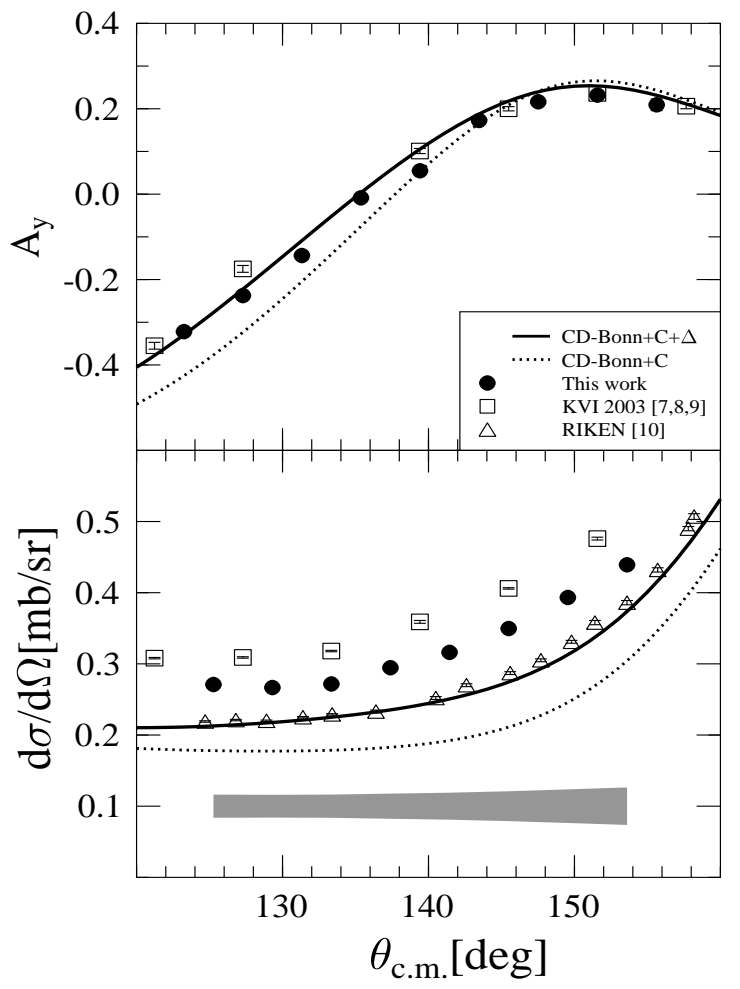

FIG. 3: The top panel shows the results for the analyzing power of the ${ }^{2} \mathrm{H}(\vec{p}, d) p$ reaction. The present work is represented by filled circles and the previously-published KVI data [7] are shown by open squares. The bottom panel shows the corresponding measurement of the differential cross section. Our results are represented by filled circles and are compared to data published in Refs. [8, 10]. The error bars represent statistical uncertainties. The width of the gray band corresponds to the systematic uncertainty of our measurement. For a description of the lines, we refer to the caption of Fig. 2

center-of-mass angle, $\theta_{\text {c.m. }}$. The error bars for all symbols represent statistical uncertainties, which, in most cases, are smaller than the symbol sizes. The measured analyzing powers, shown in the top panel of Fig. 3. are compared to previously-published KVI data from Ref. 7] (open squares). The two data sets are in very good agreement and the comparison gives confidence in the quality of our data apart from an overall normalization. Note, however, the striking discrepancy between the previously-published differential cross sections measured at KVI 8] (open squares) and RIKEN [10] (open triangles) as shown in the bottom panel of Fig. 3. The systematic uncertainties of the cross section measurements are not shown in the figure and are $2 \%$ for the RIKEN data, $4.5 \%$ for the previously-published KVI data, and $6 \%$ for the data discussed in this paper. Our results fall between these two data sets and differ significantly from both of the previous measurements. The solid and dashed lines represent the results of the Faddeev calculations by the Hannover-Lisbon theory group. The RIKEN data set would imply that the theoretical description of this three-nucleon process is well understood whereas, the KVI data indicate that not all the ingredients are incorporated yet in the models. The observed discrepancy between data and theory could originate from the fact that the predictions are solutions of the non-relativistic Lippmann Schwinger equations, which might imply that not all relativistic effects are included consistently.

The observed inconsistency for the differential cross

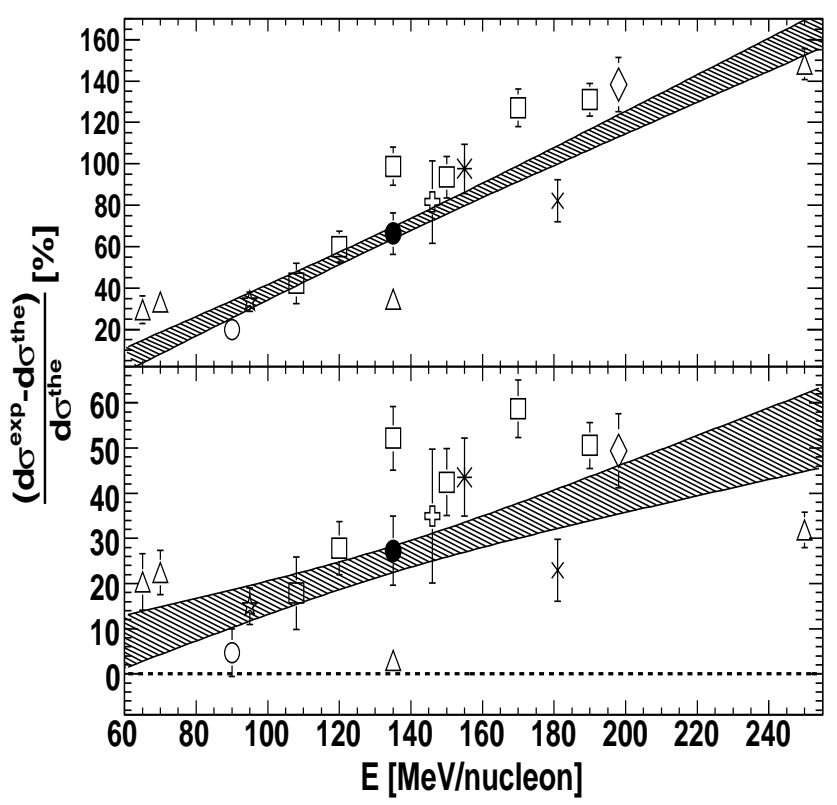

FIG. 4: The relative difference between the calculations by the Hannover-Lisbon theory group and the measured cross sections for the elastic $p+d$ reaction as a function of beam energy for $\theta_{c . m .}=140^{\circ}$. The top panel shows the differences with a calculation based on the CD-Bonn potential and the Coulomb interaction, whereas for the bottom panel also an additional $\Delta$ isobar has been taken into account. Open squares are data from [8], open triangles are data from Refs. 10, 11, 21, 22], open circle is from [14], open star is from [16], cross is from [17], star is from 15], open cross is from [13], diamond is from [18] and the filled circle is from this work. The shaded band represents the result of a line fit through the data excluding the results obtained at KVI, RIKEN and RCNP. The width of the band corresponds to a $2 \sigma$ error of the fit.

section, as shown in Fig. 3. initiated a discussion within the nuclear-physics community on the reliability of the experimental data and on how to interpret the data in terms of underlying physics, such as $3 \mathrm{NP}$ effects. It is, therefore, of importance to review these observations with respect to three-nucleon scattering data taken at other energies and in other channels. The most generic approach would be to perform a partial-wave analysis (PWA) of all available three-nucleon scattering data similar to what has been done for the nucleon-nucleon scattering data by the Nijmegen group [4]. Such an analysis would provide an independent judgment of the quality of the data sets. At present, a PWA in 
the three-nucleon sector is still a technical challenge which, so-far, has not been pursued and which is outside the scope of this paper. Instead, we have carried out a systematic study of the energy dependence of all available cross sections in elastic proton-deuteron scattering with respect to state-of-the-art calculations by the Hannover-Lisbon theory group. One example from this study is presented in Fig. 4. The top panel shows the relative difference between the model predictions excluding the $\Delta$ isobar contribution and data taken at a fixed center-of-mass angle of $\theta_{\text {c.m. }}=140^{\circ}$. The data points were extracted from a polynomial fit through each angular distributions. The error bars correspond to a quadratic sum of the statistical and systematic uncertainties of each measurement. We have made a straight-line fit through the data, excluding the measurements performed at KVI, RIKEN, and RCNP. Including these data in the fit will, however, not change the conclusions. The shaded band shows the fit function including its errors, which are calculated based on the correlation matrix of the fit parameters. Note the rapidly increasing discrepancy with energy between data and the predictions based on a high-quality two-nucleon potential. At $135 \mathrm{MeV} /$ nucleon the deviation is already more than $50 \%$, which is supported by our data. Based on the RIKEN data one would conclude that the large deviation can be resolved at $135 \mathrm{MeV} /$ nucleon by including the $\Delta$-isobar effect, as demonstrated in the bottom panel of Fig. 4. However, if this is true, it would imply that almost all experiments must suffer from a normalization problem. A similar study has been done for other center-of-mass angles, $\theta_{c . m}$. between $125^{\circ}$ and $155^{\circ}$, leading to the same observation and conclusion.

In conclusion, we have analyzed the observed discrepancy between two experimental data sets of the differential cross sections in elastic $\vec{p}+d$ scattering at $135 \mathrm{MeV}$, which were obtained in the past at RIKEN and at KVI. For this, we have remeasured the differential cross section at center-of-mass angles between $125^{\circ}$ and $155^{\circ}$, and compared our results with previouslypublished data. Furthermore, the differential cross section and analyzing powers of the inverse reaction, $\vec{d}+p$ at a beam-energy of $65 \mathrm{MeV} /$ nucleon have been obtained and compared to the existing and well-established data-base at these relatively low energies. The data for this reaction are found to be in excellent agreement with the existing data base, which proves that BINA and our analysis procedure are well suited to measure the elastic channel with high precision. The measured differential cross section for the elastic reaction at $135 \mathrm{MeV}$ differs significantly from the previously-published data taken at RIKEN and at KVI. We have carried out a systematic study of the energy dependence of all available cross sections in elastic proton-deuteron scattering. The results of this study revealed that our data consistently follow the interpolated energy dependence, whereas, in particular, the RIKEN data deviate significantly from the expected trend.

The authors acknowledge the work by the cyclotron and ion-source groups at KVI for delivering high-quality polarized beams. We thank Frans Mul for the mechanical construction of BINA and the Hannover-Lisbon group for providing the theoretical calculations. This work is part of the research program of the "Stichting voor Fundamenteel Onderzoek der Materie" (FOM) with financial support from the "Nederlandse Organisatie voor Wetenschappelijk Onderzoek" (NWO). Furthermore, the present work has been performed with financial support from the University of Groningen and the Gesellschaft für Schwerionenforschung mbH (GSI), Darmstadt. The Indiana co-authors were supported by US NFS grant PHY-0457219.
[1] R. Machleidt et al., Phys. Rev. C 53, 1483(R) (1996).

[2] R. B. Wiringa et al., Phys. Rev. C 29, 1207 (1984).

[3] V. G. J. Stoks et al., Phys. Rev. C 47, 512 (1993).

[4] M. C. M. Rentmeester et al., Phys. Rev. Lett. 82, 4992 (1999).

[5] H. Primakoff et al., Phys. Rev. 55, 1218 (1939).

[6] R. Bieber et al., Phys. Rev. Lett. 84, 606 (2000).

[7] K. Ermisch et al., Phys. Rev. Lett. 86, 5862 (2001).

[8] K. Ermisch et al., Phys. Rev. C 68, 051001(R) (2003).

[9] K. Ermisch et al., Phys. Rev. C 71, 064004 (2005).

[10] H. Sakai et al., Phys. Rev. Lett. 84, 5288 (2000).

[11] K. Sekiguchi et al., Phys. Rev. C 65, 034003 (2002).

[12] K. Sekiguchi et al., Phys. Rev. Lett. 95, 162301 (2005).

[13] H. Postma and R. Wilson Phys. Rev. 121, 1129 (1961).

[14] H. Amir-Ahmadi et al., Phys. Rev. C 75, 041001(R) (2007).

[15] K. Kuroda et al., Nucl. Phys. 88, 33 (1966).

[16] P. Mermod et al., Phys. Rev. C 72, 061001(R) (2005).

[17] G. Igo et al., Nucl. Phys. A 195, 33 (1972).
[18] R. E. Adelberger and C. N Brown Phys. Rev. D 5, 2139 (1972).

[19] H. Mardanpour et al., Eur. Phys. J. A 31, 383 (2007).

[20] E. Stephan et al., Phys. Rev. C 76, 057001 (2007).

[21] H. Shimizu et al., Nucl. Phys. A 382, 242 (1982).

[22] K. Hatanaka et al., Eur . Phys. J. A 18, 293 (2003).

[23] E. J. Stephenson et al., Phys. Rev. C 60, 061001 (1999).

[24] W. Glöckle et al., Phys. Rep. 274, 107 (1996).

[25] H. Witała et al., Phys. Rev. Lett. 81, 1183 (1998).

[26] S. Nemoto et al., Phys. Rev. C 58, 2599 (1998).

[27] M. Volkerts et al., Nucl. Instrum. and Meth. in Phys. Res. A 428, 432 (1999).

[28] N. Kalantar-Nayestanaki, J. Mulder, and J. Zijlstra Nucl. Instrum. and Meth. in Phys. Res. A 417, 215 (1998).

[29] R. Bieber et al., Nucl. Instrum. and Meth. in Phys. Res. A 457, 12 (2001).

[30] H. R. Kremers et al., Nucl. Instrum. and Meth. in Phys. Res. A 516, 209 (2004). 